\title{
Las representaciones sociales como base para el diseño de una secuencia de aprendizaje sobre el concepto de función
}

\author{
Bertha Ivonne Sánchez Luján \\ (Instituto Tecnológico de Ciudad Jiménez. México) \\ Alberto Camacho Ríos \\ (Instituto Tecnológico de Chihuahua II. México) \\ (Dirección General de Educación Superior Tecnológica. México)
}

\section{Resumen}

Presentamos el diseño y aplicación de una secuencia de aprendizaje para la construcción de una representación alternativa que permite a los estudiantes la adquisición del concepto de función mediante los significados asociados de variable, variación y variabilidad, así como la creación de un vínculo entre los distintos modos de representación de una función, con el fin de que puedan coordinarlos durante la resolución de problemas. El estudio se llevó a cabo sujetándolo a la Teoría de las Representaciones Sociales, la cual tiene como eje central las prácticas y las representaciones sociales (RS). Durante la aplicación, no fue nuestra intención romper con las (RS) que los estudiantes poseen sobre el concepto de función, sino incorporar a ésta la noción de variación. Se produjo un fenómeno de evolución progresiva de los elementos periféricos al integrar nuevos elementos sobre los anteriores, particularmente bajo la influencia del cambio de las prácticas en el aula. En lo que respecta al sistema central, se mantuvo, lo que no asombra dada su estabilidad y su resistencia al cambio. Estos resultados demuestran y confirman el impacto que las prácticas sociales ejercen sobre las concepciones que se poseen de un objeto en particular.

\section{Palabras clave}

Función - Elementos periféricos - Representaciones sociales - Sistema central - Variabilidad.

\section{Summary}

We present the design and application of a sequence of learning for the construction of an alternative representation that allows the students the acquisition of the concept of function by means of the associate meanings of variable, variation and variability. The study was realized under the theory of the Social Representations, which has as backbone the practices and the social representations (SR). During the 
application, it was not our intention to break with the (SR) that the students possess on the concept of function, but to incorporate into this one the notion of variation. Was produced a phenomenon of progressive evolution of the peripheral elements on having integrated new elements on the previous ones, particularly under the influence of the change of the practices in the classroom. Regarding the central nucleus, there was kept, which does not amaze given its stability and its resistance to the change. These results demonstrate and confirm the impact that the social practices exercise on the conceptions that are possessed of an object especially.

\section{Key words}

Function - Peripheral system - Social representations - Central system - Variability.

\section{Introducción}

El constante problema de entender del concepto de función, como una relación entre variables, ha suscitado desde el siglo pasado diversas investigaciones a su alrededor (p. e, Sierpinska, 1992; Ruiz, 1998; Guzmán, 1998). En este sentido es que nos interesamos en las concepciones de los profesores y estudiantes del nivel superior de ingeniería.

El proyecto se inició esencialmente a partir de un análisis cognitivo del concepto de función, para el que hicimos uso de la "Teoría de las Representaciones Sociales" (TRS). Los resultados nos condujeron al establecimiento de "cadenas de significados".

Por otro lado, realizamos un análisis epistemológico y un estudio del currículo escolar, los cuales no se presentan en este artículo y dieron pie al diseño de una situación desde la perspectiva variacional del propio concepto.

Los autores que han estudiado las RS insisten en su carácter construido y estructurado. Según Johsua y Dupin (1993), podemos nombrar a la representación como el contenido estructurado del pensamiento de un sujeto (citado en Dollo, 2001, p. 74), las RS no sólo son visiones del mundo, también son verdaderas reconstrucciones mentales.

En la construcción del conocimiento debe tenerse en cuenta que los estudiantes poseen representaciones previas y es la evolución progresiva de éstas lo que las lleva a un nivel operativo cercano a la realidad y proporciona herramientas para resolver problemas. Las concepciones en proceso de estructuración cognitiva evolucionan y apoyan el pensamiento que se está construyendo (Giordan y De Vecchi, 1988). Es el profesor, quien a través del discurso y las prácticas, apoya a la formación del nuevo concepto. De aquí la importancia de las prácticas que se realizan en el aula y fuera de ella.

Tal como lo predice la TRS, los elementos periféricos tienen una función de defensa, mas pueden ser cambiados (removidos, modificados, aumentados) bajo el efecto de una modificación en las prácticas sociales, lo cual, de 
acuerdo con Flament (1994), tiene como consecuencia un cambio gradual de la representación, su desintegración o transformación total. En nuestro caso, la intención fue la de incluir elementos de carácter variacional en la noción de función, para lo cual contemplamos las condiciones para la transformación de una RS propuestas por (Guimelli, 1993), a saber:

1. Que sea un evento característico con alto grado de implicación en el grupo.

2. Que contemple las circunstancias externas a la representación, entendidas como las características físicas, económicas o el ambiente social, en relación con el objeto de representación, como consecuencia del evento anterior, lo que cambia las prácticas tradicionales y su pertinencia.

3. Que el cambio de las prácticas sea percibido por el grupo como irreversible, lo que los lleve a reorganizar el campo de la representación.

Los resultados del análisis cognitivo, junto con los resultados del análisis epistemológico y del análisis didáctico (Sánchez y Camacho, 2007) nos permiten proponer una secuencia de aprendizaje, en la que integraremos en el diseño la noción de "variabilidad", que hemos reconocido en el dominio de prácticas sociales: procedimentales y de observación, que ocurrieron a lo largo de los siglos XVIII y XIX, la cual nos permitirá re-significar el concepto de función. Con este significado asociado al propio concepto, intentaremos influir en los elementos periféricos de la cognición de los estudiantes, al colocarlos en un proceso de deconstrucción y recontextualización del concepto, tal como se muestra más adelante.

\section{La Teoría de las Representaciones Sociales (TRS)}

El concepto de RS fue introducido en 1961 a partir de la teoría psicoanalítica de Freud, por Serge Moscovici en su libro "La psychanalyse, son image et son publique", en tanto que la teoría se ha desarrollado a lo largo de este tiempo. En su obra, Moscovici señala que el concepto de RS tiene dos aspectos básicos para su definición. Por un lado, son una forma de conocimiento y, por otro, son una forma de reconstrucción mental de la realidad, de tal suerte que sólo se dan en el intercambio de información con otras personas, por lo que una RS no es una respuesta a un estímulo u objeto exterior sino la reconstrucción de ese estímulo del objeto real (Moscovici, 1985). En tal sentido las RS no son estáticas, sino que se encuentran en continua variación o movimiento.

Una RS es aquélla construida por las interacciones de un grupo social y está formada por ideas, creencias, opiniones e incluso actitudes sobre algún concepto o noción en particular. La función principal de las RS es interpretar la realidad, manteniendo relaciones de simbolización y atribuyéndole significaciones (Guimelli, 2004).

Toda RS posee dos componentes:

1. "La cognitiva", pues supone un sujeto activo, y 
2. "La social", pues las prácticas sociales están determinadas por las condiciones socioculturales en que la representación se realiza y se transmite (Abric, 1994).

Las RS se conforman a partir de la información, la experiencia, el conocimiento y los modelos de pensamiento y se construyen mediante imágenes, sistemas y categorías sobre un elemento en particular:

"La representación social es una modalidad particular del conocimiento, cuya función es la elaboración de los comportamientos y la comunicación entre los individuos. (...) es un corpus organizado de conocimientos y una de las actividades psíquicas gracias a las cuales los hombres hacen inteligible la realidad física y social, se integran en un grupo o en una relación cotidiana de intercambios" (Farr, 1984, p. 496).

Luego, y para nuestro propósito, entendemos las RS como una construcción personal que integra elementos y que se ha generado en la práctica del profesor. Estas RS transforman e impactan las concepciones de los estudiantes al momento de impartir su clase mediante procedimientos y actividades en el aula.

\section{Estructura de las representaciones:}

\section{sistema central y elementos periféricos}

Toda representación está construida alrededor del sistema central, que es el elemento más resistente al cambio y es determinado tanto por la naturaleza del objeto representado, como por la relación que el grupo (o sujeto) mantiene con el objeto, y además con un sistema de valores y normas sociales. La identificación del sistema central es determinante para conocer el objeto propio de la representación. Está vinculado con la memoria colectiva y con la historia del grupo, es estable, coherente y rígido, resistente al cambio y poco sensible al contexto inmediato.

Alrededor del sistema central se tienen los "elementos periféricos" o "sistema periférico". Como elementos jerarquizados desempeñan un papel esencial en la representación, puesto que son la interfase entre el sistema central y el objeto mismo. Los elementos periféricos permiten la integración de las experiencias individuales, lo que soporta la heterogeneidad del grupo, son, además, evolutivos y sensibles al contexto inmediato. Aseguran la protección del sistema central.

Los elementos periféricos son el vínculo entre el sistema central y la situación concreta donde se elabora o trabaja la representación.

Valero (2003) presentó un estudio sobre la estabilidad y cambio de las concepciones alternativas de los estudiantes sobre el concepto de función, en el que se menciona que existen concepciones que se encuentran más arraigadas 
en los estudiantes, que son más difíciles de modificar. Desde el punto de vista de las RS, esto ocurre cuando aquellas concepciones que se quieren modificar en el sujeto, se encuentran en el sistema central de dicha representación. Las concepciones alternativas que se remueven con mayor facilidad serán, entonces, las que se encuentren en los elementos periféricos de la representación social. El mismo estudio muestra que estas concepciones alternativas sufren transformaciones, pues no pueden permanecer indefinidamente en los estudiantes, aunque sean resistentes al cambio. Nosotros agregamos que no pueden permanecer indefinidamente en los estudiantes, puesto que están vinculadas con prácticas que van a modificar la RS que de ellas se tiene. Esta modificación puede darse, incluso, tanto en el sistema central como en los elementos periféricos.

Las RS surgen en el contexto de lo familiar que opera como base para comparar y ayuda a entender lo que ocurre, y lo desconocido, que nos descontrola y nos hace representar nuevos objetos. Ese nuevo objeto se traslada entre ambos espacios y se reconstruye hasta llegar a ser familiar de nuevo.

Así, en el contexto de la enseñanza:

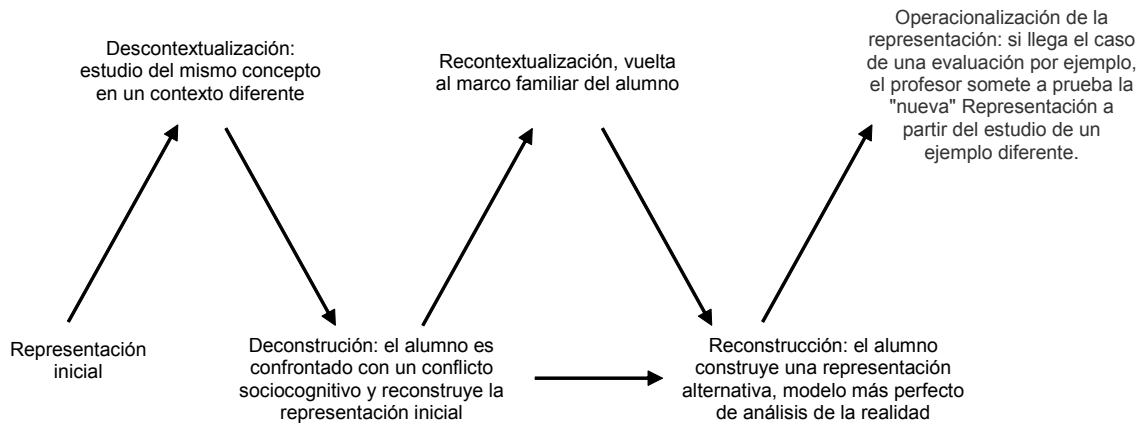

Figura 1. Estrategias didácticas del profesor y el proceso de aprendizaje de los alumnos.

Según Beitone et al. (2004; citado en Dollo y Parayre, 2005):

a) El alumno posee una representación inicial del objeto.

b) Descontextualización: El concepto es estudiado o aplicado en otra situación diferente a la anterior.

c) Deconstrucción: Cuando el alumno realiza interacciones se genera un conflicto sociocognitivo que lo lleva a reorganizar sus concepciones anteriores integrando nuevos elementos surgidos de la propia confrontación.

d) Recontextualización: Puede provenir de interacciones interindividuales o intraindividuales, y es en este momento que discute de nuevo sus propias representaciones. 
e) Reconstrucción: Construye una nueva representación que involucra una reorganización debida a las interacciones.

f) Operacionalización: Es la aplicación de la nueva representación en un caso o ejemplo diferente.

Para cambiar una RS es necesario romper con la representación anterior, lo que algunas veces resulta difícil por la coexistencia que se da entre la concepción anterior y la nueva que se proponga. En la enseñanza de la matemática, esto será posible en la medida en que se diseñen secuencias de aprendizaje que involucren a los alumnos en procesos de cambio: "(...) el conocimiento matemático aparece pues como una construcción social, por el hecho de que la base del conocimiento matemático (el conocimiento lingüístico con sus convenios y sus reglas) es una construcción social y el hecho de que los procesos sociales interpersonales de diálogo y de crítica son necesarios para convertir el conocimiento matemático subjetivo de un individuo en un conocimiento objetivo socialmente aceptado (...)" (Dubois, 2000).

El proceso ya citado será fundamental para el diseño de la situación de aprendizaje, en tanto no deseamos romper con la RS que los estudiantes poseen sobre el concepto de función, sino más bien, incorporar a ésta la noción de variación en la forma que se discute más adelante.

\section{Diseño y aplicación de la secuencia}

Al tratar las concepciones de una forma "inductiva", bastaría con desestabilizar las concepciones iniciales de los alumnos, presentando experiencias o hechos contrarios a estas ideas. Sin embargo, esto no es suficiente para inducir la necesidad de un cambio conceptual (Johsua y Dupin, 1993). El tomar en consideración las RS que los estudiantes poseen sobre el concepto de función es de gran importancia en la aplicación de la secuencia de trabajo que planteamos en este artículo para el desarrollo del tema.

El término RS hace referencia a los productos y a los procesos que caracterizan el pensamiento práctico, elaborado a través de la interacción social entre los miembros de un grupo; durante esta interacción, al tener en cuenta las concepciones de los alumnos, es posible no sólo favorecer la emergencia de un conflicto sociocognitivo, sino también la construcción de una representación alternativa, más cercana con nuestra realidad (para nosotros, se presentará tomando en cuenta la cadena de significados variable - variación - variabilidad). De esta forma, al terminar la reconstrucción hay que operacionalizarla, es decir, hacerla funcionar en la solución de nuevos problemas.

La secuencia de aprendizaje propuesta se rige bajo estas bases e ilustra las estrategias didácticas del profesor, además, toma en cuenta los procesos de aprendizaje de los estudiantes, con el fin de lograr un verdadero cambio conceptual. 
Dollo (2001) propone un proceso de discusión de opiniones, donde el profesor debe diseñar una situación que simule las condiciones de un debate "científico" vía la construcción de un saber. Johsua y Dupin (1993) señalan que no se puede imponer, o hacer construir "naturalmente" un modelo "correcto". El diseño de la secuencia debe ser tal que permita a los estudiantes formular hipótesis explicativas (aún contradictorias), bajo un proceso de conjeturarefutación, que emergen en la fase de sensibilización y se apoyan sobre sus propias concepciones. Algunas de estas hipótesis verbalizadas pueden ser eliminadas por los propios alumnos en su discusión que, guiados por el profesor, les permitirá llegar a la fase de reconstrucción.

\section{El concepto de función a través de las nociones de variable - variación - variabilidad}

El diseño de la secuencia de aprendizaje propuesta tiene como eje central la noción de variabilidad, la cual emerge de una práctica social ejercida en las prácticas de ingeniería. De esta manera, incorporamos un registro ausente en la enseñanza actual del propio concepto de función, con el objetivo de hacer patente la variación en el discurso desde esta etapa.

Los conceptos de variable, variación y variabilidad están involucrados en el concepto de función, mas, en la actualidad se dejan de lado para la adquisición del concepto por parte de los estudiantes, creándose así un "hueco" en su entendimiento (Camacho, 2006).

La secuencia presenta estas tres nociones que permiten el estudio del movimiento de los fenómenos desde diversos modos de representarles: geométrico, algebraico y variacional.

Objetivo: Permitir a los estudiantes la adquisición del concepto de función mediante los significados asociados, como son: variable, variación y variabilidad, así como la creación de un vínculo entre los distintos modos de representar una función, con el fin de que puedan coordinarlos durante la resolución de problemas.

La secuencia consta de cinco fases, divididas en actividades: la primera Fase de Sensibilización, cuyo objetivo es hacer emerger las concepciones de los estudiantes y ponerlos en discusión sobre ellas para formular cierto número de hipótesis relativas a los criterios necesarios para la definición de variable y variación; incluye la Actividad 1 donde el profesor inicia con la descripción del movimiento de algunos objetos matemáticos como son el punto, la recta y el plano. De ahí sigue la Actividad 2, donde se presenta el comportamiento de las variables al mover uno de los lados de un triángulo.

En la Fase de Descontextualización (aquélla en la que el concepto es estudiado o aplicado en otra situación diferente a la anterior), se realiza un debate y desestabilización de las representaciones, el profesor pide mediante 
una lluvia de ideas grupales que se discuta la relación entre las variables a través del aumento o de la disminución de la altura en un trapecio.

En la Fase 3: Deconstrucción (cuando el alumno es confrontado con un conflicto sociocognitivo y reconstruye la representación inicial), se pide una expresión analítica que muestre el fenómeno, además es aquí donde se enuncia el concepto de función como una dependencia de cantidades variables, incluye además una segunda actividad por equipos de 3 o 4 personas para coordinar los distintos modos de representar una función.

La cuarta Fase de Reconstrucción (El alumno construye una representación alternativa, modelo más perfecto de análisis de la realidad), presenta dos actividades de vinculación entre los diversos modos de representar una función, siendo ésta la etapa donde el alumno debe construir una representación alternativa, que involucre los elementos mostrados.

Para terminar, la quinta Fase Operacionalización (el profesor pone a prueba la "nueva" representación, por ejemplo en una evaluación), se compone de dos actividades de exploración y verificación en las que los estudiantes deberán aplicar las nociones vistas y responder preguntas encaminadas a lograr una conexión entre los modos de representación en los casos presentados, para, finalmente, proporcionar una definición del concepto de función que involucre los significados asociados incorporados en la situación.

Las fases de deconstrucción y construcción de una concepción alternativa, en este diseño son presentadas sobre la base del debate en equipo y luego grupal. Durante las actividades de trabajo en equipos, el profesor debe circular entre los mismos y pedir a los alumnos precisión en sus respuestas y justificación de éstas.

Existen fenómenos de descontextualización y recontextualización sucesivos en cada etapa de reorganización de la información. Las prácticas sociales sucesivas como son la selección de los contenidos de enseñanza, o el diseño de secuencias por el profesor, incluyen reconstrucciones de un objeto nuevo $y$, en éstas, debe tomarse en cuenta que los estudiantes no llegan a las aulas como un libro en blanco, sino que poseen todo un conjunto de representaciones sobre los objetos a tratar (Giordan y De Vecchi, 1995).

Una representación puede considerarse como un modelo personal de organización de los conocimientos con relación a un problema particular en un contexto definido. No podemos hablar de la representación como de la noción en sí. La diferencia entre representación y concepto científico no es una diferencia de grado, sino constituyen dos modos distintos de conocimiento.

\section{Descripción de la situación de aprendizaje}

Fase 1. Sensibilización.

Objetivo: Emergencia de las representaciones de los alumnos. 
Actividad 1. Introducción.

El profesor pide a los alumnos que imaginen el movimiento de ciertos objetos como el punto, la recta, la superficie. Tal como se ejemplifica enseguida:

En el pizarrón se muestra cómo una línea recta es creada por el movimiento de un punto.

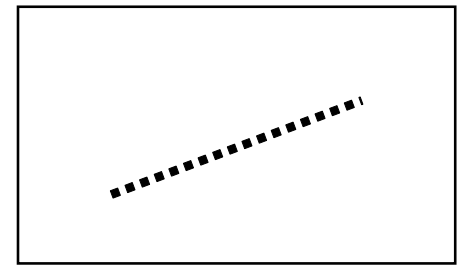

Figura 2

La línea recta, al moverse describe una superficie.

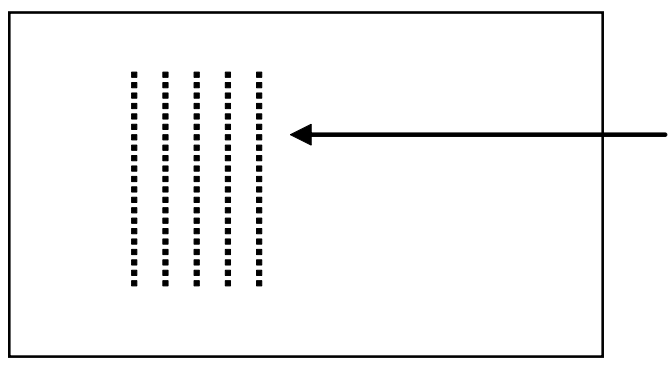

Línea en movimiento

Figura 3

La superficie al moverse describe un sólido.

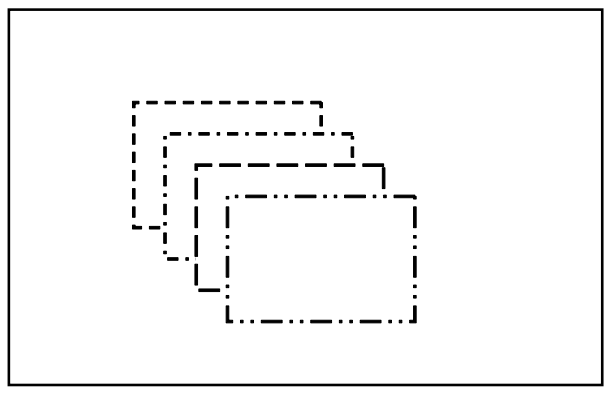

Figura 4

La cualidad principal de las variables es que representan el movimiento de los fenómenos físicos y geométricos que se estudian a través del cálculo diferencial. 
Actividad 2. Variación de la variable.

El triángulo $A B C$ se encuentra en estado de constancia o reposo:

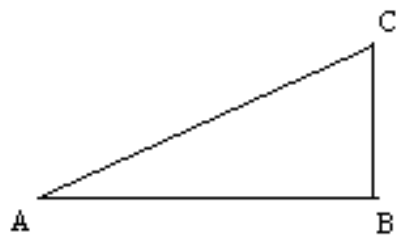

Figura 5

En el siguiente ejercicio se muestra la variación de uno de los lados del triángulo $A B C$.

Si damos la oportunidad al lado BC de moverse hacia arriba, el mismo se convierte en una variable.

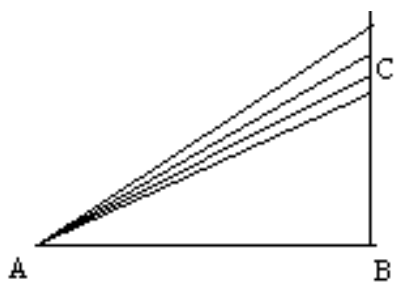

Figura 6

Cada uno de estos movimientos son llamados instantáneas y producen variación. Algunos de ellos se muestran enseguida.
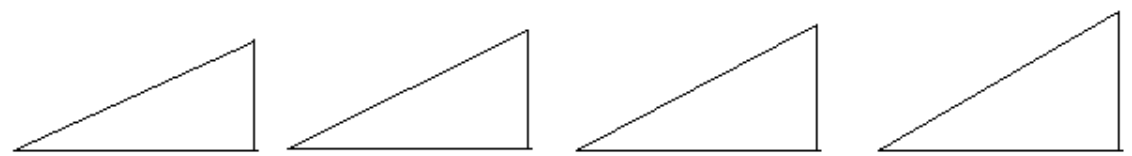

Figura 7

El lado AB se dice que está fijo o en "estado de constancia".

"Todo lo constante se dice que está fijo".

Al dejar de moverse posee de nuevo un valor de constante o reposo.

En el siguiente ejemplo, la imagen representa el movimiento de un cohete lanzado al espacio, el cual es observado por una persona colocada a una distancia a del lanzamiento. El primer cambio que sufre la figura con el movimiento del cohete es una variación o instantánea del propio movimiento. Una instantánea es como una fotografía tomada en determinado momento de una de las variaciones del suceso. 


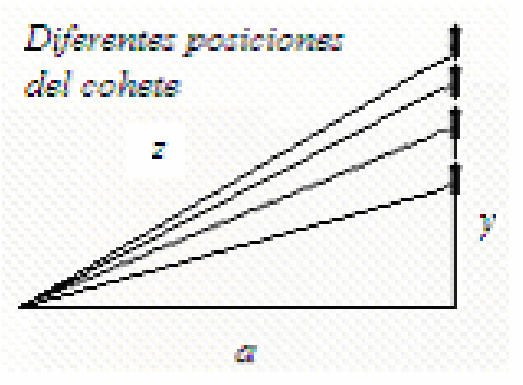

Figura 8

Preguntas: Para cada una de las instantáneas: ¿Cómo es el área? ¿Es la misma? ¿Es diferente?

Fase 2. Descontextualización.

Objetivo: Reafirmar la relación entre variables.

Actividad 1.

En esta fase se presentan otros ejemplos que apoyen la fase anterior.

Consideremos que tenemos un trapecio $A B C D$, donde $A B$ es la base menor y $C D$ es la base mayor, ambas constantes, si hacemos que la altura aumente o disminuya:

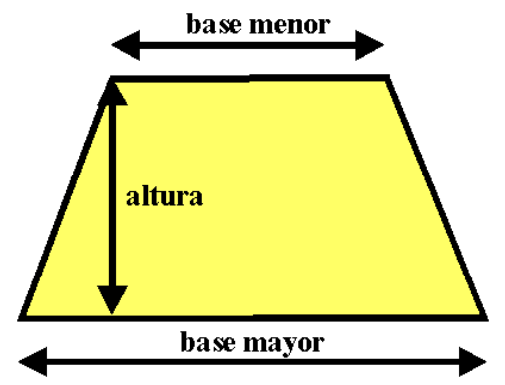

Figura 9
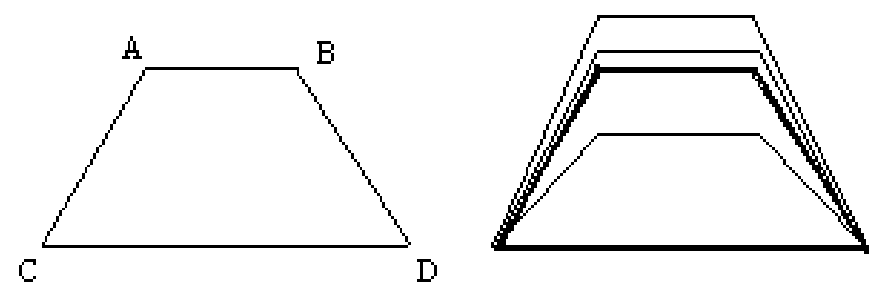

Figura 10 
Cada uno de los movimientos es una instantánea:
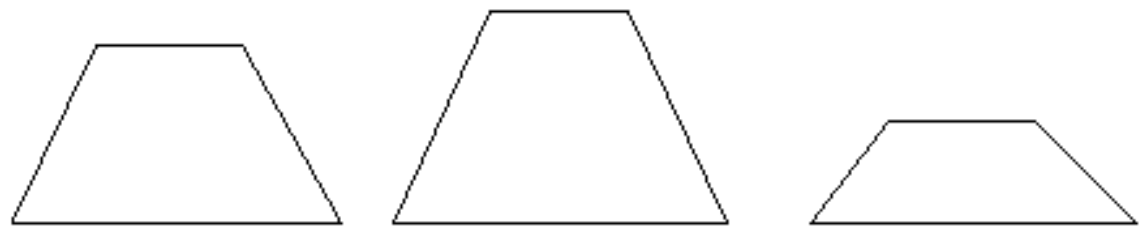

Figura 11

De manera grupal y mediante una lluvia de ideas, los alumnos responden las siguientes preguntas:

Para cada una de las instantáneas: ¿Cómo es el área? ¿Es la misma? ¿Es diferente? ¿De qué depende que cambie el área?

Lo cierto es que en el primer ejemplo cambiaron las longitudes $B C$ y $A C$, el área $A=\frac{a}{2}$ del triángulo (donde a representa la base e $y$ la altura).

Así mismo, en el segundo caso los lados $A C$ y $B D$, el área $A=\frac{h}{2}\left(b_{1}+b_{2}\right)$ del trapecio (donde $h$ es la altura, $b_{1}$ y $b_{2}$ las bases menor y mayor), etc.

Fase 3: Deconstrucción.

Objetivo: Confrontar a los alumnos con un conflicto sociocognitivo y reconstruir la representación inicial.

\section{Actividad 1.}

Dado que se han presentado los elementos para identificar las variables, se pide a los estudiantes que proporcionen una expresión analítica que muestre ese cambio tanto para el caso del triángulo como para el del trapecio. El profesor debe guiar la discusión en torno a las dos ecuaciones de área y concluir al respecto.

La realización de este tipo de reconocimiento geométrico de las variaciones, es la que más adelante nos permitirá el estudio analítico del propio movimiento; es decir, no consentiremos solamente en ver su expresión variacional. A la cantidad de variaciones que se pueden establecer a partir de las expresiones $A(y)=\frac{a}{2}$ y $A(h)=\frac{h}{2}\left(b_{1}+b_{2}\right)$ se le llama variabilidad, la cual representa el total de las variaciones producidas por el fenómeno. En el caso de las ecuaciones, $A=\frac{a}{2}, A=\frac{h}{2}\left(b_{1}+b_{2}\right)$, éstas significan el caso particular de una de las variaciones originadas por el fenómeno, y dejan ver al movimiento en un estado estacionario o de constancia, en el cual es factible su análisis. 
Actividad 2. Coordinación de los distintos modos de representar funciones.

Se presentan cuatro maneras de mostrar una función:

1. Verbal.

2. Tabla de valores.

3. Gráfica.

4. Algebraica.

Se ha observado que los estudiantes presentan serias dificultades en conectar esos contextos, e incluso, los perciben como modos separados (Font, 2000). En algunos casos los estudiantes pueden describir correctamente lo que sucede en un evento, otras construir una gráfica a partir de los datos observados, o resolver un problema dada su función expresada en forma analítica. Sin embargo el paso de un sistema de representación a otro causa conflictos en clase. Cuando a un estudiante se le pide que resuelva un problema el cual involucra la interpretación de datos para responder ciertas preguntas, o el obtener conclusiones a partir de una gráfica cuando no se da la expresión analítica, causa serios problemas, pues la mayoría de las veces consideran cada uno de los contextos por separado. De ahí la importancia de incluir elementos operacionales que nos ayuden a lograr esa vinculación.

Se divide el grupo en equipos de 3 o 4 personas. El profesor comenta la siguiente situación:

Usted tiene un vaso con agua fría y coloca dentro de él unos cubos de hielo luego lo deja sobre la mesa, en un día caluroso del mes de julio.

Pide a los estudiantes que respondan a lo siguiente:

a) Describan con palabras cómo cambia la temperatura del agua a medida que pasa el tiempo.

b) A continuación tracen una gráfica aproximada de la temperatura del agua como función del tiempo transcurrido.

Después de esto un representante de cada grupo pasa a exponer la solución del problema.

El profesor apoya al grupo para la conclusión del problema.

Fase 4: Reconstrucción.

Objetivo: Construir una representación alternativa.

Actividad 1.

A los mismos equipos se les proporciona el siguiente ejercicio:

La siguiente tabla muestra el registro de temperatura medido cada dos horas, desde la media noche hasta las 12 del mediodía, un día de otoño. El tiempo $t$ se midió en horas a partir de la media noche. La temperatura T viene dada en grados centígrados. 
Tabla 1

\begin{tabular}{|c|r|r|r|r|r|r|r|}
\hline$t$ & 0 & 2 & 4 & 6 & 8 & 10 & 12 \\
\hline$T$ & 15 & 14 & 12 & 10 & 11 & 14 & 16 \\
\hline
\end{tabular}

a) Trace una gráfica de T en función de $t$.

b) Describa con palabras lo que ocurre en la gráfica.

c) Utilice la gráfica para estimar la temperatura a las 5 a.m. y a las 11 a.m. Actividad 2.

Los estudiantes continúan en el mismo equipo y se les presenta el siguiente ejercicio para que llenen la tabla y respondan las preguntas:

Se tiene un globo de volumen $V=\frac{4}{3} \pi r^{3}$ cuyo radio varía al ser llenado con aire.

Tabla 2

\begin{tabular}{|c|c|}
\hline$R$ & $V$ \\
\hline 0.5 & \\
\hline 0.9 & \\
\hline 1.0 & \\
\hline 2.5 & \\
\hline 3.0 & \\
\hline 3.8 & \\
\hline
\end{tabular}

Después de llenar la tabla responde las siguientes preguntas: ¿Cómo se comporta el volumen del globo conforme se llena de aire? ¿De qué depende ese cambio?

Muestra gráficamente el cambio del volumen respecto al radio.

Escribe una expresión analítica que represente la totalidad de los cambios en función del radio.

Al final de la fase, un representante de cada grupo pasa a exponer los resultados de las dos actividades.

El profesor apoya al grupo para la conclusión del problema.

Fase 5: Operacionalización.

Objetivo: Poner a prueba la "nueva" representación mediante su aplicación en un problema específico. 


\section{Actividad 1: Exploración.}

Es de suma importancia verificar que las diversas representaciones estén presentes en la resolución de problemas, e identificar si el estudiante las vincula adecuadamente.

Un avión vuela horizontalmente tomando contacto en tierra con una torre de control en diversos momentos de tiempo antes de pasar por encima de ella, la cual se encuentra verticalmente a 10000 metros del avión.

a) Describe con palabras lo que ocurre con la distancia entre el avión y la torre conforme pasa el tiempo.

b) Desarrolla una gráfica que muestre la simulación del movimiento del avión con respecto a la torre para diferentes momentos de contacto.

c) Toma una de las instantáneas de la simulación y dibújala al lado. Puesto que es un triángulo rectángulo, nombra los lados variables con las letras $z$ y $x$, el lado constante es $10000 \mathrm{~m}$.

d) Dado que el avión está en movimiento horizontal hacia la torre, la distancia entre éstos también cambia. Establece una relación que involucre ambas variables, incluyendo la constante. (Sugerimos utilizar el Teorema de Pitágoras).

e) Con la expresión obtenida llenar la siguiente tabla:

Tabla 3

\begin{tabular}{|c|c|}
\hline $\begin{array}{c}\text { Distancia horizontal entre el } \\
\text { avión y el punto por encima } \\
\text { de la torre de control ( ) }\end{array}$ & $\begin{array}{c}\text { Distintas posiciones de la } \\
\text { distancia del avión a la torre } \\
\text { de control en tierra ( ) } \\
\text { Expresión: }\end{array}$ \\
\hline 20,000 & \\
18,000 & \\
12,000 & \\
7,000 & \\
5,000 & \\
1,000 & \\
\hline
\end{tabular}

f) Escribe una expresión analítica que represente la totalidad de los cambios.

Actividad 2.

A partir de los ejemplos anteriores, expresa con tus propias palabras el concepto de función. 


\section{Aplicación de la secuencia}

La secuencia de aprendizaje se aplicó por el mismo profesor a dos grupos de estudiantes de la materia de Cálculo Diferencial del Instituto Tecnológico de Cd. Jiménez:

Tabla 4

\begin{tabular}{|l|c|c|c|c|c|}
\hline \multicolumn{1}{|c|}{ Carrera } & Turno & $\begin{array}{c}\text { Número de } \\
\text { Participantes }\end{array}$ & $\begin{array}{c}\text { Hora de } \\
\text { Inicio }\end{array}$ & $\begin{array}{c}\text { Hora de } \\
\text { Terminación }\end{array}$ & Receso \\
\hline $\begin{array}{l}\text { Ingeniería } \\
\text { Industrial }\end{array}$ & Matutino & 20 & $11: 00$ a.m. & $1: 55$ p.m. & no \\
\hline $\begin{array}{l}\text { Ingeniería } \\
\text { Electrome- } \\
\text { cánica }\end{array}$ & Vespertino & 20 & $4: 00$ p.m. & $6: 35$ p.m. & $6: 00$ a $6: 15$ \\
\hline
\end{tabular}

Ambos grupos se dividieron en seis equipos de trabajo: dos equipos de cuatro integrantes y cuatro equipos de tres integrantes cada uno. El primer grupo en que se aplicó la secuencia fue el de la carrera de Ing. Industrial en una sola sesión de 2 horas y 55 minutos, y se pidió como evidencia que cada equipo escribiera en una hoja de rotafolio el resultado de cada actividad y pasara al frente a exponerla ante sus compañeros y la escriban de nuevo en el pizarrón; considerando que el tiempo de aplicación se alargó por esta razón, al aplicarla en el grupo de Ing. Electromecánica se les pidió que al explicarla utilizaran la misma gráfica realizada en la hoja de rotafolio, y de esta manera el tiempo se redujo a 2 horas 20 minutos.

Recomendamos que para futuras aplicaciones de ser posible se realice en tres sesiones, ya que, aun cuando el trabajo se desarrolló de manera fluida, al final de la sesión los alumnos se mostraron inquietos por la duración de la misma.

Tabla 5

\begin{tabular}{|l|c|c|c|}
\hline Fase & Actividad & $\begin{array}{c}\text { Tiempo } \\
\text { aproximado } \\
\text { (en minutos) }\end{array}$ & Comentarios \\
\hline 1. Sensibilización & $\begin{array}{c}\text { 1. Introducción } \\
\text { 2. Variación } \\
\text { de la variable }\end{array}$ & $5 \mathrm{~min}$. & $\begin{array}{c}\text { El profesor expone ante } \\
\text { el grupo y los estudiantes } \\
\text { participaron activamente } \\
\text { en la lluvia de ideas }\end{array}$ \\
\hline $\begin{array}{c}\text { 2. Descontex- } \\
\text { tualización }\end{array}$ & $\begin{array}{c}\text { 1. Reafirmar la } \\
\text { relación entre } \\
\text { variables }\end{array}$ & $10 \mathrm{~min}$. & $\begin{array}{c}\text { Se define la noción de } \\
\text { variación como: el cambio de } \\
\text { magnitud de una cantidad }\end{array}$ \\
\hline
\end{tabular}

(continúa en página 61) 
(viene de página 60)

\begin{tabular}{|l|c|c|c|}
\hline 3. Deconstrucción & $\begin{array}{c}\text { 1. Modos de } \\
\text { representación } \\
\text { 2. Resolución } \\
\text { de un problema } \\
\text { en equipo }\end{array}$ & $\begin{array}{c}5 \mathrm{~min} . \\
30 \mathrm{~min} .\end{array}$ & $\begin{array}{c}\text { Aquí finaliza la exposición por } \\
\text { parte del profesor } \\
\text { Por ser el primer ejercicio } \\
\text { tardaron más tiempo }\end{array}$ \\
\hline 4. Reconstrucción & $\begin{array}{c}\text { 1. Actividad de } \\
\text { reconstrucción } \\
\text { 2. Ejercicio por } \\
\text { equipo }\end{array}$ & $25 \mathrm{~min}$. & $\begin{array}{c}\text { En esta fase tardaron } \\
\text { menos tiempo en realizar las } \\
\text { actividades }\end{array}$ \\
\hline 5.Operacionalización & $\begin{array}{c}\text { 1. Exploración } \\
\text { 2. Concepto }\end{array}$ & $25 \mathrm{~min}$. & $\begin{array}{c}\text { Se notó un poco de dificultad } \\
\text { con los "números grandes" al } \\
\text { realizar las operaciones } \\
\text { En la definición incluyen } \\
\text { significados alternativos como: } \\
\text { "instantáneas", "cambios", } \\
\text { "movimientos" }\end{array}$ \\
\hline
\end{tabular}

\section{Resultados de la aplicación}

Fases de Sensibilización y Descontextualización:

Al preguntar ¿qué cambia? respondieron:

Tabla 6

\begin{tabular}{|c|c|}
\hline Triángulo & Trapecio \\
\hline El ángulo & Altura \\
El área & Los ángulos \\
BC & El área \\
Hipotenusa AC & Los lados \\
La forma & \\
\hline
\end{tabular}

Fase de Deconstrucción:

a) Describan con palabras cómo cambia la temperatura del agua a medida que pasa el tiempo.

b) A continuación tracen una gráfica aproximada de la temperatura del agua como función del tiempo transcurrido. 
Tabla 7

\begin{tabular}{|c|c|c|}
\hline Equipo & Grupo 1 & Gráfica \\
\hline 1 & $\begin{array}{c}\text { El agua en el vaso sigue igual cuando } \\
\text { introducen los hielos pero sigue pasando } \\
\text { el tiempo aumenta la temperatura del } \\
\text { agua y los hielos se derriten }\end{array}$ & $\begin{array}{l}\text { Triángulo donde uno de los catetos } \\
\text { es temperatura del agua y tiempo } \\
\text { en la hipotenusa, la cual "varía" }\end{array}$ \\
\hline 2 & $\begin{array}{c}\text { El agua pierde calor mientras que el } \\
\text { hielo gana calor, la temperatura va } \\
\text { descendiendo cada vez más lento en } \\
\text { el agua hasta llegar a un punto cero } \\
\text { y comienza a ganarle el calor el frío y } \\
\text { después el agua toma la temperatura } \\
\text { ambiente }\end{array}$ & $\begin{array}{l}\text { En las abscisas la temperatura } \\
\text { ambiente, la curva inicia en cero } \\
\text { y desciende }\end{array}$ \\
\hline 3 & $\begin{array}{l}\text { Al momento de colocar los hielos en el } \\
\text { agua al momento de que se derriten la } \\
\text { temperatura del agua aumenta en sí se } \\
\text { vuelve más fría y su volumen aumenta } \\
\text { cuando se derriten los hielos y al } \\
\text { transcurso de cierto tiempo con el calor } \\
\text { del medio ambiente la temperatura del } \\
\text { agua cambia se vuelve más caliente }\end{array}$ & $\begin{array}{l}\text { La temperatura inicia en }-5 \text { grados, } \\
\text { desciende hasta }-20 \text { y luego } \\
\text { sube hasta } 35 . \text { Nombra el eje de } \\
\text { temperatura más no el de tiempo }\end{array}$ \\
\hline 4 & $\begin{array}{l}\text { Llega un momento en que el agua se } \\
\text { pone fría luego empieza a ponerse } \\
\text { caliente el agua conforme pasa el tiempo }\end{array}$ & $\begin{array}{l}\text { La temperatura inicia en } 8 \\
\text { disminuye sin llegar a cero para } \\
\text { luego subir. No nombra los ejes }\end{array}$ \\
\hline 5 & $\begin{array}{c}\text { La temperatura del agua va } \\
\text { disminuyendo conforme el hielo se } \\
\text { fusiona (pasa de sólido a líquido) a } \\
\text { temperatura ambiente. Pero cuando se } \\
\text { derrite el hielo en el agua, tiende a ir } \\
\text { aumentando la temperatura }\end{array}$ & $\begin{array}{l}\text { Muestra una curva suave que } \\
\text { inicia en } 30^{\circ} \text {, baja y sube de } \\
\text { nuevo. Nombra eje de tiempo }\end{array}$ \\
\hline 6 & $\begin{array}{l}\text { Si el agua está fría y se le pone hielo el } \\
\text { agua se pone más fría, conforme que el } \\
\text { tiempo pasa los hielos se van derritiendo } \\
\text { poco a poco y el agua se va calentando } \\
\text { hasta llegar a temperatura ambiente }\end{array}$ & $\begin{array}{l}\text { Inicia con una curva que } \\
\text { desciende y luego sube hasta } \\
\text { llegar a una línea constante }\end{array}$ \\
\hline
\end{tabular}

Los tres primeros equipos grafican por debajo de la línea de cero, los dos siguientes grafican sin considerar que al final la temperatura deberá permanecer constante. Notamos que los alumnos no están acostumbrados a describir verbalmente un evento. 
Tabla 8

\begin{tabular}{|c|c|c|}
\hline Equipo & Grupo 2 & Gráfica \\
\hline 1 & $\begin{array}{c}\text { La temperatura va a decrecer de manera } \\
\text { constante con respecto a la temperatura } \\
\text { inicial debido al intercambio de calor, } \\
\text { al derretirse el hielo por completo, el } \\
\text { agua tendrá una temperatura más baja, } \\
\text { después de este cambio la temperatura } \\
\text { ascenderá hasta igualar la temperatura } \\
\text { ambiente }\end{array}$ & $\begin{array}{c}\text { Nombra los dos ejes } \\
\text { correctamente y muestra una } \\
\text { curva suave que concuerda con la } \\
\text { explicación textual }\end{array}$ \\
\hline 2 & $\begin{array}{l}\text { Primero llegará a una temperatura fría } \\
\text { máxima. Después empezará a derretir } \\
\text { el hielo y empezará poco a poco a } \\
\text { aumentar la temperatura del agua } \\
\text { hasta llegar al punto en que el hielo se } \\
\text { convierta en agua completamente y si } \\
\text { se sigue así empezará a evaporar el } \\
\text { agua por la temperatura }\end{array}$ & $\begin{array}{l}\text { Nombra los ejes como "grados"y } \\
\text { "minutos", inicia la gráfica en } \\
\text { el origen la cual sube de forma } \\
\text { casi lineal hasta los } 30^{\circ} \text { y luego } \\
\text { permanece constante }\end{array}$ \\
\hline 3 & $\begin{array}{l}\text { La temperatura baja cuando se le } \\
\text { colocan los hielos, después va tomando } \\
\text { la temperatura del medio ambiente hasta } \\
\text { que llega a la temperatura del medio y } \\
\text { empieza a evaporarse en poca proporción }\end{array}$ & $\begin{array}{c}\text { No nombra los ejes. Inicia en } 10^{\circ} \\
\text { desciende y luego sube hasta } \\
\text { llegar a una constante }\end{array}$ \\
\hline 4 & $\begin{array}{c}\text { La temperatura del agua fría con hielos } \\
\text { empieza a cambiar a medida que } \\
\text { empieza a adquirir la temperatura del } \\
\text { medio ambiente que lógicamente es } \\
\text { más caliente que la del agua, conforme } \\
\text { transcurre el día comienzan a tener un } \\
\text { equilibrio térmico. En pocas palabras } \\
\text { el líquido llega a tomar la temperatura } \\
\text { ambiente }\end{array}$ & $\begin{array}{l}\text { Intercambia los ejes. Inicia en } \\
\text { el origen y va aumentando la } \\
\text { temperatura para luego descender }\end{array}$ \\
\hline 5 & $\begin{array}{c}\text { La temperatura del agua es más alta y la } \\
\text { del hielo es más baja, cuando éstas se } \\
\text { mezclan llegan a un punto igual }\end{array}$ & No sabe cómo graficarlo \\
\hline 6 & $\begin{array}{l}\text { El agua a temperatura ambiente, al } \\
\text { momento que se le echa el hielo empieza } \\
\text { a bajar la temperatura, llega a su menor } \\
\text { temperatura. Conforme avanza el tiempo } \\
\text { luego el agua llega a un momento en que } \\
\text { la temperatura empieza a subir hasta que } \\
\text { llega un momento que la temperatura del } \\
\text { agua se pone a temperatura ambiente }\end{array}$ & $\begin{array}{l}\text { Grafica correctamente de acuerdo } \\
\text { a lo descrito }\end{array}$ \\
\hline
\end{tabular}


* En la trascripción se respetaron las palabras originales, corrigiéndose solamente la ortografía.

Se observa dificultad para expresar el evento.

En cuanto a las gráficas se percibe la influencia numérica, es decir, la mayoría intenta colocar números a pesar de que en el ejemplo no se especifican.

Fase de Reconstrucción:

Actividad 1.

En esta actividad encontramos que todos los equipos de ambos grupos realizaron correctamente la gráfica, describieron acertadamente el evento y los valores obtenidos para la temperatura a las 5 a.m., fue de $11^{\circ}$ y para las 11 a.m., de $15^{\circ}$. Concluimos que, por ser un ejemplo del todo numérico (a lo cual los alumnos están habituados) no presentó mayor dificultad.

Ejemplos de descripciones:

\section{Tabla 9}

\begin{tabular}{|c|c|c|}
\hline $\begin{array}{l}\text { En las } 12 \text { de la media } \\
\text { noche es más alta } \\
\text { y en la madrugada } \\
\text { como } 5 \text { y } 6 \text { de la } \\
\text { mañana desciende. } \\
\text { Conforme avanzan } \\
\text { las horas vuelve a } \\
\text { aumentar }\end{array}$ & $\begin{array}{c}\text { La temperatura } \\
\text { disminuye de la } \\
\text { 0:00 hrs. a las } 6 \\
\text { a.m. hasta } 5^{\circ} \mathrm{y} \\
\text { posteriormente } \\
\text { incrementa de las } \\
\text { 6:00 a.m. a las 12:00 }\end{array}$ & $\begin{array}{c}\text { La temperatura disminuye al paso de } \\
\text { las primeras } 2 \text { horas en un grado, a las } \\
2 \text { horas disminuye } 2 \text { grados más, para } \\
\text { las otras dos horas disminuye otros } \\
\text { dos grados, en las siguientes } 2 \text { horas } \\
\text { aumentó } 1 \text { grado, en las otras } 2 \text { horas } \\
\text { aumentó } 3 \text { grados y en las últimas dos } \\
\text { horas aumentó } 2 \text { grados }\end{array}$ \\
\hline
\end{tabular}

\section{Actividad 2.}

Se tiene un globo de volumen $V=\frac{4}{3} \pi r^{3}$ cuyo radio varía al ser llenado con aire.

En esta etapa los resultados de la tabla son correctos en todos los equipos en ambos grupos y asimismo lo son las respuestas a las preguntas formuladas. En cuanto a la expresión analítica, la mayoría la escribió de forma correcta.

\section{Tabla 10}

\begin{tabular}{|c|c|c|}
\hline $\begin{array}{c}\text { ¿Cómo se comporta el volumen } \\
\text { del globo conforme } \\
\text { se llena de aire? }\end{array}$ & ¿De qué depende ese cambio? & $\begin{array}{c}\text { Expresión } \\
\text { analítica }\end{array}$ \\
\hline $\begin{array}{c}\text { Grupo 1 } \\
\text { Va aumentando el volumen } \\
\text { El volumen va aumentando }\end{array}$ & $\begin{array}{c}\text { Aire } \\
\text { De la cantidad de aire } \\
\text { Del radio }\end{array}$ & $V=\frac{4}{3} \pi r^{3}$ \\
$V(r)=\frac{4}{3} \pi r^{3}$
\end{tabular}




\begin{tabular}{|c|c|c|}
\hline $\begin{array}{l}\text { Conforme el radio aumenta } \\
\text { Va creciendo respecto al radio } \\
\text { El volumen va aumentando } \\
\text { El volumen del globo se va } \\
\text { expandiendo }\end{array}$ & $\begin{array}{c}\text { El radio } \\
\text { Porque el radio cambia conforme } \\
\text { se va llenando de aire } \\
\text { Depende del radio del globo }\end{array}$ & $\begin{aligned} V & =n \pi(r)^{n} \\
V & =\frac{4}{3} \pi r^{3} \\
V & =\frac{4}{3} \pi r^{3} \\
V(r) & =\frac{4}{3} \pi r^{3}\end{aligned}$ \\
\hline $\begin{array}{c}\text { Grupo } 2 \\
\text { El volumen aumenta } \\
\text { considerablemente con respecto } \\
\text { al radio } \\
\text { Va aumentando el volumen cúbico } \\
\text { Va aumentando conforme se llena } \\
\text { Aumenta } \\
\text { Incrementa } \\
\text { Que el volumen va aumentando }\end{array}$ & $\begin{array}{c}\text { Del radio (incremento) } \\
\text { Debido a la presión del aire } \\
\text { introducido } \\
\text { De la cantidad de aire que entra y } \\
\text { lógicamente aumenta su diámetro y } \\
\text { su volumen } \\
\text { Del aumento del radio } \\
\text { Del aumento del radio } \\
\text { Depende del radio del globo }\end{array}$ & $\begin{array}{l}V(r)=\frac{4}{3} \pi r^{3} \\
V(R)=\frac{4}{3} \pi r^{3} \\
V(r)=\frac{4}{3} \pi r^{3} \\
V=(r) 4.1887 r^{3} \\
V(r)=\frac{4}{3} \pi r^{3} \\
V(r)=\frac{4}{3} \pi r^{3}\end{array}$ \\
\hline
\end{tabular}

Fase de Operacionalización:

Actividad 1: Exploración.

Tabla 11

\begin{tabular}{|c|c|}
\hline $\begin{array}{c}\text { Distancia horizontal entre el } \\
\text { avión y el punto por encima } \\
\text { de la torre de control ( })\end{array}$ & $\begin{array}{c}\text { Distintas posiciones de la } \\
\text { distancia del avión a la torre } \\
\text { de control en tierra ( ) } \\
\text { Expresión: }\end{array}$ \\
\hline 20,000 & $22,360.67 \mathrm{~km}$. \\
18,000 & $20,591.26$, \\
12,000 & $15,620.44$ \\
7,000 & $12,206.55$ \\
5,000 & $11,180.33$ \\
1,000 & $10,049.87$ \\
\hline
\end{tabular}

f) Escribe una expresión analítica que represente la totalidad de los cambios. 
Resultados:

Tabla 12

\begin{tabular}{|c|c|c|c|c|c|}
\hline Grupo 1 & Gráfica & Triángulo & Tabla & Relación & Expresión \\
\hline $\begin{array}{l}\text { 1. Varía, ya que antes } \\
\text { de pasar por la torre las } \\
\text { distancias } x, z \text { disminuyen, } \\
\text { y al pasar la torre las } \\
\text { distancias aumentan }\end{array}$ & Bien & Bien & $z, x$ & $\begin{array}{l}\text { Bien. Sin } \\
\text { unidades }\end{array}$ & $\begin{array}{l}c^{2}(b)=a^{2}+b^{2} \\
x^{2}(z)=a^{2}+z^{2}\end{array}$ \\
\hline 2. Disminuye & Bien & Bien & $z, x$ & Bien. Km. & $z(x)=\sqrt{x^{2}+y^{2}}$ \\
\hline $\begin{array}{l}\text { 3. Aumenta la hipotenusa } \\
\text { entre la torre y el avión }\end{array}$ & Bien & Bien & $\mathrm{z}, \mathrm{x}$ & $\begin{array}{l}\text { Bien. Sin } \\
\text { embargo } \\
\text { nombra } \\
\text { b,c a los } \\
\text { lados }\end{array}$ & $c=\sqrt{a^{2}+b^{2}}$ \\
\hline $\begin{array}{c}\text { 4. Aumenta } \\
\text { horizontalmente }\end{array}$ & Bien & Bien & $\mathrm{z}, \mathrm{x}$ & Bien & $z=\sqrt{a^{2}+x^{2}}$ \\
\hline 5. Disminuye & Bien & Bien & $z, x$ & Bien & $c^{2}(a)=a^{2}+b^{2}$ \\
\hline $\begin{array}{l}\text { 6. La distancia del avión } \\
\text { va disminuyendo en el eje } \\
\text { x llega un momento que } \\
\text { la distancia en el eje x } \\
\text { del avión y de la torre de } \\
\text { control es de cero. Pero la } \\
\text { distancia entre el avión y la } \\
\text { torre de control en el eje x } \\
\text { vuelve a aumentar }\end{array}$ & Bien & Bien & $\mathrm{z}, \mathrm{x}$ & Bien. Km & $z(x)=\sqrt{x^{2}+y^{2}}$ \\
\hline Grupo 2 & Gráfica & Triángulo & Tabla & Relación & Expresión \\
\hline 1. Constante & Bien & Bien & $\mathrm{z}, \mathrm{x}$ & Incorrecto & $x=\sqrt{z^{2}+c^{2}}$ \\
\hline $\begin{array}{l}\text { 2. La distancia vertical no } \\
\text { cambia, sólo la distancia } \\
\text { horizontal la cual se va } \\
\text { reduciendo conforme } \\
\text { avanza el avión }\end{array}$ & Bien & Bien & $z, x$ & Bien & $x(z)=\sqrt{10000^{2}+z^{2}}$ \\
\hline 3. Va disminuyendo & Bien & Bien & $z, x$ & Incorrecto & $c^{2}(x)=a^{2}+b^{2}$ \\
\hline 4. El avión se va acercando & Bien & Bien & $z, x$ & Incorrecto & $A(x)=\sqrt{a^{2}+b^{2}}$ \\
\hline
\end{tabular}

(continúa en página 67) 
(viene de página 66)

\begin{tabular}{|c|c|c|c|c|c|}
\hline $\begin{array}{c}\text { 5. La distancia horizontal } \\
\text { se hace más corta } \\
\text { mientras que la vertical } \\
\text { queda igual }\end{array}$ & Bien & Bien & $\mathbf{z}, \mathbf{x}$ & Bien & $\begin{array}{c}c(z)=\sqrt{a^{2}+b^{2}} \\
z=\sqrt{x^{2}+10000^{2}}\end{array}$ \\
\hline $\begin{array}{c}\text { 6. La distancia del avión y } \\
\text { la torre se van acortando } \\
\text { conforme pasa el tiempo y } \\
\text { después de determinado } \\
\text { tiempo la distancia vuelve } \\
\text { a crecer }\end{array}$ & Bien & Bien & z, x & Bien & $x(z)=\sqrt{10000^{2}+z^{2}}$ \\
\hline
\end{tabular}

En la solución de este problema los puntos a), b), c) y d) fueron resueltos en forma correcta por la mayoría de los equipos, la única diferencia son las literales asignadas con cada uno de los lados del triángulo.

Actividad 2.

A partir de los ejemplos anteriores, expresa con tus propias palabras el concepto de función.

Tabla 13

\begin{tabular}{|c|c|}
\hline EQUIPO & GRUPO 1 \\
\hline 1 & $\begin{array}{l}\text { Una función es una expresión analítica que se utiliza para resolver } \\
\text { las variaciones (dependencias) de un problema tomando como } \\
\text { base una ecuación }\end{array}$ \\
\hline 2 & Sirve para darle resultado a una variable \\
\hline 3 & $\begin{array}{l}\text { Se refiere a cuando se tienen diversas figuras del mismo tipo pero } \\
\text { diferentes dimensiones se escribe área de un lado para decir que } \\
\text { va a aumentar esa cifra }\end{array}$ \\
\hline 4 & $\begin{array}{l}\text { Una función sirve para calcular los cambios de un ejercicio, con una } \\
\text { misma ecuación, agregándole lo que varía }\end{array}$ \\
\hline 5 & No respondieron \\
\hline \multirow[t]{2}{*}{6} & $\begin{array}{l}\text { Una función sirve para calcular puntos instantáneos de objetos en } \\
\text { movimiento }\end{array}$ \\
\hline & GRUPO 2 \\
\hline 1 & Es algo que nos ayuda a sacar el total de todos los movimientos \\
\hline 2 & $\begin{array}{c}\text { Una función }(x) \text { y se representa con ciertas variables y llegar a la } \\
\text { resolución de un problema }\end{array}$ \\
\hline
\end{tabular}




\begin{tabular}{|c|c|}
\hline 3 & $\begin{array}{r}\text { Es una forma de representar la totalidad de los cambios. Forma de } \\
\text { representar una ecuación que incluya todo }\end{array}$ \\
\hline 4 & $\begin{array}{r}\text { Es algo que nos sirve para resolver algo mediante procesos. Es } \\
\text { cómo se representa ciertas variables para llegar a una solución de } \\
\text { un problema }\end{array}$ \\
\hline 5 & $\begin{array}{r}\text { Es cuando una variable depende de otra variable. Una incógnita } \\
\text { que se tiene que buscar en base a una expresión }\end{array}$ \\
\hline 6 & $\begin{array}{r}\text { La función es la totalidad de un fenómeno, se toma en cuenta } \\
\text { diferentes instantes del fenómeno lo cual describe los cambios } \\
\text { que ocurren conforme pasa el tiempo del fenómeno. Es como una } \\
\text { fórmula que sirve para cualquier situación que se presente, sirve } \\
\text { para diferentes instantes }\end{array}$ \\
\hline
\end{tabular}

En esta última parte notamos un cambio en la RS del concepto de función por parte de los estudiantes, entendidas éstas como las concepciones que poseen del propio concepto, ya que las respuestas son dadas en términos de "algo que cambia", tal como se manejó durante la aplicación de la secuencia.

\section{Conclusiones finales}

Para el diseño de la secuencia de aprendizaje es necesario recuperar las RS que un individuo posee sobre cierto concepto para, a partir de ello y a través del diseño, guiarlo en una práctica que permita reconstruir (según el proceso de aprendizaje presentado anteriormente) la representación que tiene del concepto. Durante la aplicación del diseño no fue nuestra intención romper con las RS que los estudiantes poseen sobre el concepto de función, sino incorporar a ésta la noción de variación. Puesto que las RS son definidas a través del sistema central, que como planteamos posee una gran resistencia al cambio, entonces los elementos periféricos tienen un papel fundamental en el análisis de los procesos que originan la dinámica de una RS. En un estudio realizado por Sánchez (2009), sobre las RS que los estudiantes poseían acerca del concepto de función, encontró un sentido de dependencia de variables, influenciadas por profesores, los propios libros de texto.

La aplicación de esta secuencia de trabajo propuesta produjo un fenómeno de evolución progresiva de los elementos periféricos al integrar nuevos elementos sobre los anteriores, particularmente bajo la influencia del cambio de las prácticas en el aula. En lo que respecta al sistema central, se mantuvo, lo que no asombra dada su estabilidad y su resistencia al cambio.

En este sentido, consideramos que en la RS en torno al concepto de función, los estudiantes integraron en los elementos periféricos la noción de 
variablilidad, ya que en la práctica, al pedirles que resolviesen un problema, encontramos procedimientos que dan muestra de ello, al aplicar los "nuevos" contenidos de estas representaciones. En particular en el problema del avión que sobrevuela una torre de control, ellos utilizan en la gráfica varias "instantáneas" y en la mayoría de los casos representan acertadamente el dibujo que los lleva a la resolución del problema, lo que confirma los elementos de la representación.

Las respuestas del último punto indican como "algo que muestra la totalidad de los cambios", "lo que varía", "diferentes instantes", en cuyo discurso está presente la noción de variación.

Estos resultados demuestran y confirman el impacto que las prácticas sociales ejercen sobre las concepciones que se poseen de un objeto en particular.

El aplicar la TRS representó un reto importante para nuestra propuesta, ya que las investigaciones hasta ahora realizadas no incorporan su manejo a objetos matemáticos en sí, sino a lo que se piensa acerca de ellos, o a la aplicación de alguna metodología en clase de matemáticas (Dubois y Dagau, 1999); o al impacto que tiene una RS sobre un proceso matemático en un aula de matemáticas con alumnos de diferentes nacionalidades (Abreu y Gorgorió, 2007); o como las RS que posee el profesor sobre sus alumnos en un aula multicultural de matemáticas las cuales incluyen dimensiones individuales como son necesidades, motivación, intereses, lugar de aprendizaje y capacidades, e influyen en la manera de impartir la clase (Santesteban, 2006). En nuestra investigación, demostramos que es posible la aplicación de esta teoría para el rescate de las concepciones que se tienen de un concepto matemático y que es posible aplicar el modelo que la propia TRS proporciona para la transformación de una representación, adaptándolo al diseño de una situación de aprendizaje en la que se toman en cuenta tanto las RS, como los resultados del análisis preliminar al rescatar un concepto que se ha perdido en la propia evolución de la enseñanza o que no es tomado como eje principal para la construcción de la noción de un concepto.

\section{Referencias Bibliográficas}

- Abreu, G. y Gorgorió N. (2007) Social representations and multicultural mathematics teaching and learning. Working group 10: Mathematics education in multicultural settings. CERME 5, pp.1559-1566.

- Abric, J. C. (1994) Pratiques sociales et représentations. Paris, PUF.

- Camacho, A. (2006) Revisión de las prácticas sociales y la socioepistemología. México, Educación Matemática No. 18 (1), pp. 133-160.

- Dollo, Ch. (2001) Quels déterminants pour l'évolution des savoirs scolaires en Sciences Economiques et Sociales? (l'exemple du chômage). Tesis de doctorado. U. de Provence, Francia. 
- Dollo, Ch. y Parayre, S. (2005) Et l'amour dans tout ça ? Des conceptions des élèves à la construction de savoirs scientifiques sur la familla. Numéro spécial de la revue Skholê IUFM de l'academie d’Aix-Marseille, pp. 41-63.

- Dubois, L. y Dagau, P-Ch. (1999) Le travail de groupe dans les nouveaux moyens de math 1P: attitudes d'enseignants. Tesis de Licenciatura en Ciencias de la Educación. Université de Genève. Obtenido de http://tecfa.unige. ch/ laurent/didact/memoire.htm el 1 de febrero de 2007.

- Dubois, L. (2000) Les modèles de l'apprentissage et les mathématiques. Obtenido de http://tecfa.unige.ch/ laurent/didact/theories.htm el 4 de febrero de 2007.

- Farr, R. (1984) Psicología social II. Pensamiento y vida social. Psicología social y problemas sociales. En S. Moscovici (Ed.). Las representaciones sociales. Barcelona, Paidós, pp. 495-506.

- $\quad$ Flament, C. (1994) "Pratiques et représentations sociales". En J. Abric (Ed.) Représentations sociales et pratiques. Paris, PUF.

- Font, V. (2000) Algunos puntos de vista sobre las representaciones en didáctica de las matemáticas. Representation in mathematics education.

- Giordan, A. y De Vecchi, G. (1988) Los orígenes del saber. De las concepciones personales a los conocimientos científicos. España, Diana Editora.

- $\quad$ Giordan, A. y De Vecchi, G. (1995) Los nuevos modelos de aprendizaje ¿más allá del constructivismo? Perspectivas No. 25(1), pp. 65-72.

- Guimelli, Ch. (1993) Concerning the structure of social representations. Papers on Social Representations - Testes sur les Représentations Sociales. Vol. 2 (2), pp. 85-92.

- Guzmán, I. (1998) "Registros de representación, el aprendizaje de nociones relativas a funciones: voces de los estudiantes". Revista Latinoamericana de Investigación en Matemática Educativa, Vol. $1 N^{\circ}(1)$, pp. 5-21.

- Joshua, S. y Dupin (1993) Introduction á la didactique des sciences et des mathématiques. Paris, PUF.

- Moscovici, S. (1985) Psicología Social l y ll. Barcelona, Paidós.

- Ruiz, L. (1998) La noción de función: análisis epistemológico y didáctico. España, Publicaciones de la Universidad de Jaén.

- Sánchez, B. I. (2009) El concepto de función matemática entre los docentes a través de las representaciones sociales. Tesis doctoral no publicada, CICATA-IPN.

- Sánchez, B. I. y Camacho, A. (2007) El concepto de función matemática en los docentes a través de las representaciones sociales. XII Conferencia Interamericana de Educación Matemática. CIEM. México.

- Santesteban, M. (2006) Representaciones sociales del profesor de matemáticas acerca de los alumnos inmigrantes: un primer Master Thesis, Programa de Doctorat en Didàctica de les Ciències i les matemàtiques. Barcelona, UAB. 
- $\quad$ Sierpinska, A. (1992) "On understanding the notion of function". En E. Dubinsky \& G. Harel (Eds). The concept of function: Aspects of epistemolofy and pedagogy. Washimgton, DC, USA: Mathematical Association of America. pp. 25-28.

- Valero, M. (2003) Estabilidad y cambio de concepciones alternativas acerca de funciones en situación escolar. Tesis de maestría no publicada. CICATA IPN. México. 